Sādhanā Vol. 36, Part 6, December 2011, pp. 995-1008. (C) Indian Academy of Sciences

\title{
A finite element model for independent wire rope core with double helical geometry subjected to axial loads
}

\author{
CENGIZ ERDONMEZ $^{1, *}$ and C ERDEM IMRAK ${ }^{2}$ \\ ${ }^{1}$ Istanbul Technical University, Institute of Informatics, Computational Science and \\ Engineering Program, 34469 Maslak, Istanbul, Turkey \\ ${ }^{2}$ Istanbul Technical University, Faculty of Mechanical Engineering, \\ 34394 Gumussuyu, Istanbul, Turkey \\ e-mail: cerdonmez@gmail.com; imrak@itu.edu.tr
}

MS received 27 March 2009; revised 26 March 2010; accepted 1 June 2010

\begin{abstract}
Due to the complex geometry of wires within a wire rope, it is difficult to model and analyse independent wire rope core accurately (IWRC). In this paper, a more realistic three-dimensional modelling approach and finite element analysis of wire ropes are explained. Single helical geometry is enough to model simple straight strand while IWRC has a more complex geometry by inclusion of double helical wires in outer strands. Taking the advantage of the double helical wires, three-dimensional IWRCs modelling is applied for both right regular lay and lang lay IWRCs. Wire-bywire based results are gathered by using the proposed modelling and analysis method under various loading conditions. Illustrative examples are given for those show the accuracy and the robustness of the present FE analysis scheme with considering frictional properties and contact interactions between wires. FE analysis results are compared with the analytical and available test results and show reasonable agreement with a simpler and more practical approach.
\end{abstract}

Keywords. Wire strand; independent wire rope core; double helices; wire-by-wire analysis.

\section{Introduction}

On the design of wire ropes, a simple straight strand is used as a core strand and it is wound by outer strands to complete the whole geometry. Independent wire rope core (IWRC) is a special type of wire rope, which can be used in applications and becomes the core for some other types of ropes known as Seale IWRC or Warrington IWRC. Large tensile force strength of the wire ropes is very important in application areas wherein the small bending and torsional stiffness. In general, two types of lay construction are used in wire rope strands: regular lay and lang lay.

*For correspondence 
In regular lay wire rope constructions, lay directions of the wires within the outer strand are opposite in direction to the core strand. In lang lay wire rope constructions, on the other hand, the directions of the wires are the same as the direction of the core strand.

Under axial loading conditions, regular lay wire ropes tend to tighten up while lang lay ropes have the tendency to unwind; for this reason lang lay ropes are used only when rope ends are restrained from rotating. When the wire rope is run under large lateral compressive loads and when additional axial loading capacity is required IWRCs are preferred (Velinsky 1989).

Wire rope theory is based on a well-known classical treatise on elasticity by Love (1944) and general nonlinear equilibrium equations are derived and presented. It is found that the frictionless theory is widely used to solve equilibrium equations because of the nonlinear behaviour and the complex nature of the wire ropes.

Although considerable research studies have been carried out about the analytical solutions of IWRCs by Costello \& Sinha (1977), Costello (1990), Velinsky et al (1984), Velinsky (1985), Velinsky (1989), Jolicoeur \& Cardou (1991), Elata et al (2004), Usabiaga \& Pagalday (2008), a little work has been done using the double helical geometry and real modelling approaches via numerical analysis. All the above-mentioned analytical models takes into account a simple straight strand as being the main component for modelling IWRC and more complex wire ropes by using IWRC.

A frictionless theory is presented for the determination of the static response of a simple straight strand in Costello \& Sinha (1977) which was the one of the first analytical solutions using the theory of Love (1944). Costello (1990) has also presented the general behaviour of the wire ropes in different aspects such as static response of reduced rope rotation, simplified bending theory for wire rope.

A wire rope with complex cross sections as a Seale IWRC is analysed in a manner, which will predict the axial static response by Velinsky et al (1984). A general nonlinear theory to analyse complex wire rope is developed as an extension of the frictionless strand theory of Costello for a Seale IWRC by Velinsky (1985). Wire ropes with three types of cores: independent wire rope cores (IWRC), fibre-core (FC) and wire strand core (WSC) are investigated and Velinsky (1989) developed a design methodology for multi-lay wire strands. A number of analytical models of twisted wire ropes under axisymmetric loads are compared simultaneously with each other and with the test results in Jolicoeur \& Cardou (1991). Among them Phillips \& Costello's (1985) model is remarkable which represents excellent correlation with the available experimental results in the literature. It is emphasized that the evaluation of the local effects such as interwire or interlayer pressure and nonlinear behaviour should be undertaken while comparing to more advanced models.

Complex structures such as IWRC are solved using rod theory and the nonlinear equilibrium equations of Love (1944) by taking the core strand as a straight wire and outer six strands as six single helical wires and assuming the whole system as a simple straight strand by using homogenization hypothesis.

However, Elata et al (2004) proposed a new model simulating the mechanical response of a wire rope with an IWRC for open sieves that emulate a well-lubricated rope, and closed sieves that emulate infinite friction between adjacent wires, which fully consider the double-helix configuration of individual wires within the IWRC. In the literature, the advantage of wire-by-wire modelling approach is introduced on the basis of the general thin rod theory and compared with the fibre models in Usabiaga \& Pagalday (2008). Most of the theoretical analyses over IWRCs are relied on some kind of homogenization hypothesis except the theoretical studies in Elata et al (2004) and Usabiaga \& Pagalday (2008). During the wire rope model subjected to axial loads, above-mentioned models are considered and a new finite element model is proposed. 
Finite element approach has been embedded in wire rope analysis since 1999. An early approach was the termination of the end effects of a wire strand, which models $0.2 \%$ of a pitch length. It also showed the frictional effects of a simple straight strand by Jiang \& Henshall (1999). Then a concise finite element model which took full advantage of the helical symmetry features of a simple straight strand is developed by Jiang et al (1999). Jiang et al (2000) has also extended their study to three-layered straight helical wire ropes under axial loads. Nawrocki \& Labrosse (2000) which takes into account every possible interwire motion have developed a finite element model of a simple straight strand based on a Cartesian isoparametric formulation.

A three-dimensional finite element model of an optical ground wire is composed to predict the stress distribution in each component when the cable is subjected to a given elongation. Modelling considerations are discussed using ADINA software for one and two layers of metallic single helical wires wound around a core tube containing optical fibres. Results of stress analysis are presented in some detail for two examples; six wires and ten wires wound around a central tube. Analyses with different cable lengths without friction for six-wire strand model are carried out for various pitches, and the one-pitch cable length was selected. For a length of one pitch, it has been shown by the authors that the stress state is almost uniform in a cross-section at half pitch length of the cable. The problem with the shorter lengths was that they could not develop adequate contact between the wires and the tube. It has been presented by Fekr et al (1999) that the end effects are limited to few elements and end effects in helical wire are shown.

The application of the homogenization method to practical situations appears to be limited, especially by means of a numerical approach. The numerical implementation of the homogenization method is performed using finite element analysis of the basic cell. Using the homogenization procedure principle, only one three-dimensional period of the cable (one pitch length) has been modelled using Samcef FEM code by Cartraud \& Messager (2006). The geometry of the strands are generated exactly by extruding circular surfaces along the centroidal helical curves of the wires as in (Fekr et al 1999), geometrical approximation have a great influence on numerical results i.e., circular strand cross-sections instead of the real elliptical geometry (Cartraud \& Messager 2006).

The various applications of homogenization discussed in the literature have demonstrated its efficiency and usefulness for the overall modelling of beam-like structures exhibiting periodic geometrical or material heterogeneity. It is based on the asymptotic expansion method, and gives the first-order approximation of the three-dimensional heterogeneous solution. Starting from the homogenization theory of periodic slender domains and taking benefit of the property of helical symmetry, the overall elastic behaviour is obtained from the solution of three-dimensional problems posed on a reduced basic cell that allows significant reduction of the size of the numerical model by Messager \& Cartraud (2008).

Validity domain of analytical models of steel wire strands is determined by Ghoreishi et al (2007). The elastic behaviour of a simple straight strand geometry having lay angles up to $35^{\circ}$ subjected to axial static load is analysed. Nine analytical model and FE model results compared by using general dimensionless stiffness coefficients. A complete 3D FE model is used to determine the validity domain of analytical models. The influence of contact conditions (wire/core contact) is examined for two limit cases: sliding without friction and merging. It has been shown that the overall static behaviour is not sensitive to these modelling hypotheses and performed preliminary tests for models of lengths between two and ten pitches shows that end effects do not influence the overall axial response (Ghoreishi et al 2007).

The behaviour of simple wire strand with multi-contact modes is analysed using finite element model by Jiang et al (2008). Radius of helical wires is such that the helical wires are in 
contact with both core and outer wires within a strand, which forms a statically indeterminate contact problem. Using the symmetric feature of the strand, a small basic sector of $1 / 6$ of the complete cross-section with a small sector length is used. Numerical results show that contacts can occur simultaneously at all possible contact points when the strand is subjected to extension with both ends restrained to rotation. The contacts with the conventional assumptions made in the analytical models of neglecting the local contact deformation. Loading and unloading curve is presented and it has been shown that for frictional analysis the loading and unloading curve indicates that a hysteretic phenomenon exists when the strand is subjected to cyclic loads. However, the influence on the global behaviour of the strand is shown to be very small by Jiang et al (2008).

In previous studies, outer strands of IWRCs are modelled by means of some kind of homogenization hypothesis, as if, the wires were helically wrapped around the core. This phenomenon suppresses the geometrical properties of double helical trajectory of wires within an IWRC. In this paper, a new numerical method is used while geometry construction of double helical geometry of wires within an IWRC which is taken into account in the analytical models developed by Elata et al (2004) and Usabiaga \& Pagalday (2008) for IWRC. Construction issue of IWRC solid and meshed model, using definition of the double helical geometry, is presented first. Then the finite element model generation considering frictional effects and contacts between wires are explained over a more realistic 3-D solid numerical model. The benefit of this model is to obtain wire-by-wire numerical results of each wire within a simple straight wire strand and IWRC. Interactions between wires within a strand are established by definition of contacts between wires and both analytical and numerical models are compared. Analytical results of axial loading condition without rotation are investigated over an IWRC model.

\section{Modelling the independent wire rope core}

Due to its complex geometry, it is not simple and straightforward to obtain wire-by-wire stress and strain analysis results rigorously considering theoretical models. Most of the finite element based analyses carried on the modelling arc length and basic sector of a simple straight strand to see the mechanical behaviours. The present study considers a more realistic model of a wire strand and IWRC, instead of a model based on arc length or a basic sector of a simple straight strand. An IWRC, consists of a simple straight strand as a core strand and six outer strands wrapped around it, is depicted using an accurate meshed form in figure 1.

In this model, a straight wire strand composed by a straight core wire of radius $R_{1}$ and six single helical wires of radius $R_{2}$ around it as shown in figure 2(a). Helix angle $\alpha_{2}$ is determined by using the related equation of pitch length of $p_{2}$ by solving the equation $\tan \alpha_{2}=p_{2} / 2 \pi r_{2}$ using $r_{2}=R_{1}+R_{2}$.

To model the outer wires of an IWRC, outer wire geometry is treated with using special parametric equations. When the outer strand of the IWRC is considered, single helical wire is taken into account as the reference centerline for the outer double helical wires. to produce the double helical geometry of the IWRC it is necessary to formulate the centerline of the double helical geometry based on the single helical wire as a core. To do this centerline of a single helical wire is considered by using its parametric equation as,

$$
\begin{aligned}
& x_{s}=r_{s} \cos \left(\theta_{s}\right), \\
& y_{s}=r_{s} \sin \left(\theta_{s}\right), \\
& z_{s}=r_{s} \tan \left(\alpha_{s}\right) \theta_{s},
\end{aligned}
$$




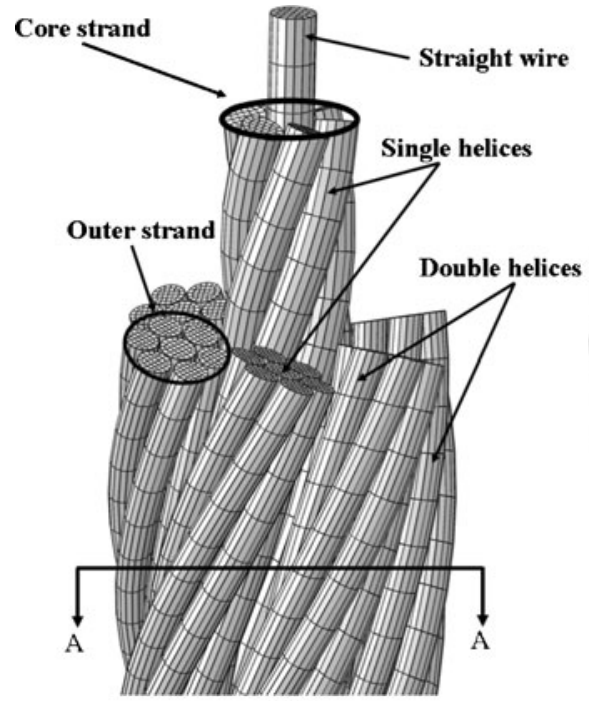

(a)

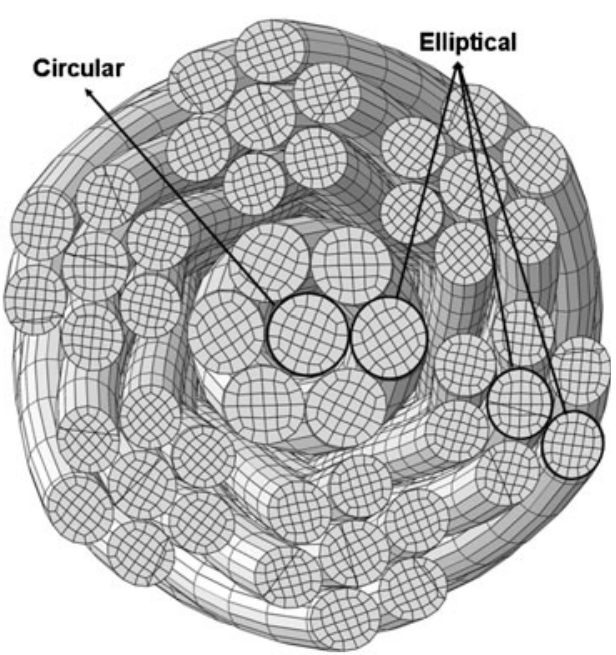

(b)

Figure 1. (a) A right lang lay $(6 \times 7)$ wire meshed IWRC. (b) A real cross sectional view of section A-A.

where $r_{s}$ is the radius of the single helix, $\alpha_{s}$ is the single helix lay angle and $\theta_{s}$ is the position of the wire within a strand. The outer double helical wires are wound around the given centerline of the single helical wire in Eqn. (1) by using the following parametric equations defined for double helical geometry,

$$
\begin{gathered}
x_{d}=x_{s}\left(\theta_{s}\right)+r_{d} \cos \left(\theta_{d}\right) \cos \left(\theta_{s}\right)-r_{d} \sin \left(\theta_{d}\right) \sin \left(\theta_{s}\right) \sin \left(\alpha_{s}\right), \\
y_{d}=y_{s}\left(\theta_{s}\right)+r_{d} \cos \left(\theta_{d}\right) \sin \left(\theta_{s}\right)+r_{d} \sin \left(\theta_{d}\right) \cos \left(\theta_{s}\right) \sin \left(\alpha_{s}\right), \\
z_{d}=z_{s}-r_{d} \sin \left(\theta_{d}\right) \cos \left(\alpha_{s}\right),
\end{gathered}
$$

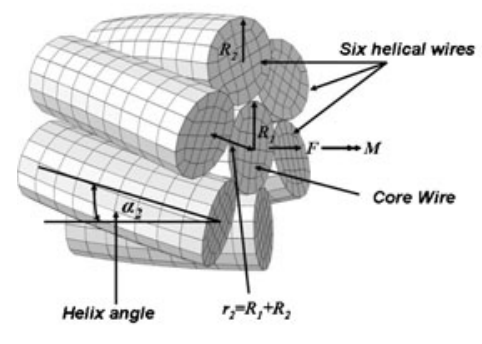

(a)

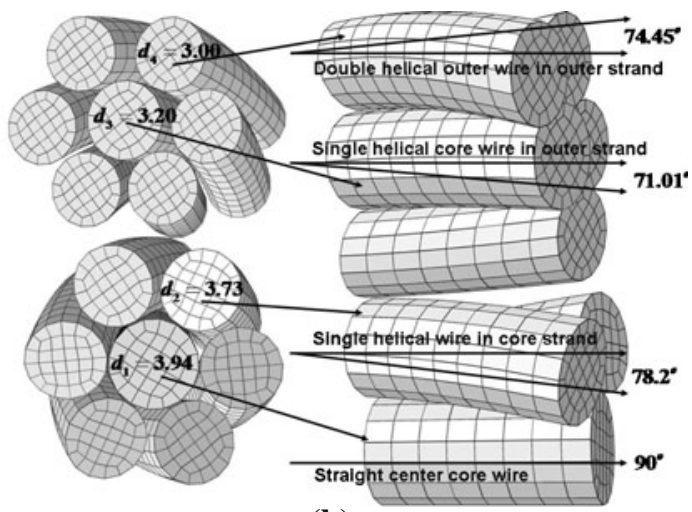

(b)

Figure 2. (a) Simple straight strand. (b) Wire lengths and helix angles of an IWRC. 
where $\theta_{d}=m \theta_{s}+\theta_{d 0}, \theta_{d 0}$ is the wire phase angle and $r_{d}$ is the distance along the double helix wire centerline and single helix strand centerline shown. The construction parameter $m$ is a constant value that can be estimated by $m=\theta_{d} / \theta_{s}$. Parametric equations used to model an IWRC and solid modelling procedure is explained by İmrak \& Erdönmez (2010). According to Eqns. (1)-(4) a right lang lay wire rope structure can be constructed and to construct a left lang lay wire rope, it is enough to negate one of the coordinate values of $x_{d}, x_{d}$ or $z_{d}$ given in Eq. (2)-(4).

\section{Numerical study of an IWRC under axial loading}

One of the important issues while modelling a wire rope strand for numerical analysis is to select a correct wire strand or wire rope length, which is used to model and analyse. Jiang \& Henshall (1999) showed that the region from 3 to 9 per cent of the pitch length is that in which both contact and sliding exist. In addition, the problem with the shorter lengths is mentioned by Fekr et al (1999) that they could not develop adequate contact between the wires and the end effects are limited to few elements. Due to these facts, wire lengths are selected within the range of effective length while the end effects are neglected during the numerical analysis.

For the simple straight strand numerical model, strand length is selected enough to guarantee contacts between center and outer wires of the strand. Pitch length of the simple straight strand is defined as $p=115 \mathrm{~mm}$. It is selected to be quarter of the pitch length of the simple straight strand as $28.75 \mathrm{~mm}$ in this study. Surface to surface contact interactions between center and six outer single helical wires and between six helical wires are defined individually. During the numerical FEA, tangential and normal contact properties are defined. Contact property of tangential behaviour with penalty frictional formulation is used with friction coefficient. The geometrical parameters of the simple straight strand are presented at the bottom of figure 2(b) and it is selected to be used as the core strand for the IWRC in the following numerical application with different pitch length.

An IWRC with $18 \mathrm{~mm}$ length $(6 \times 7)$ is considered as the second numerical application in this study. Geometrical parameters are presented for the IWRC and presented in figure 2(b). Pitch lengths of the helical wires within the IWRC are selected as follows; inner helical wire $p_{2}=70 \mathrm{~mm}$, outer center wire $p_{2}^{*}=193 \mathrm{~mm}$, double helical wire $p_{4}=70 \mathrm{~mm}$.

Material's elastic-plastic properties used are listed as; Young's modulus $E=188000 \mathrm{MPa}$, plastic modulus $E_{\mathrm{p}}=24600 \mathrm{MPa}$, yield stress $R_{\mathrm{p} 0.2}=1540 \mathrm{MPa}$, ultimate tensile stress $R_{\mathrm{m}}=$ $1800 \mathrm{MPa}$, Poisson's ratio $v=0.3$ and friction coefficient $\mu=0.115$ (Jiang \& Henshall 1999).

Quadratic hexahedral brick elements with $x, y$ and $z$ displacements DOFs are preferred to analyse nonlinear effects of the complex geometry of wire strand and IWRC. The simple straight strand and IWRC are meshed using 6496 and 18387 quadratic hexahedral elements of type C3D20R, with 32053 and 94405 nodes respectively.

The boundary conditions under axial loading are harmonious with the boundary conditions of Costello's model. One side of the boundary is constrained under fixed end boundary condition while the other end is restrained not to rotate only in $z$ directions and defined as $\Theta_{z}=0$. The axial strain of $\varepsilon=0, \ldots, 0.015$ is applied to the free end of the model for analysis. Agreements of the proposed model with other available models are shown by comparisons.

A code is developed by the first author to build the wire geometry in Matlab ${ }^{\mathrm{TM}}$ which generates the control points of helical geometries using parametric equations of both single and double helices given in Eq. (2)-(4). All kinds of wire geometry generations are brought together by writing a GUI interface code named as Wire Rope Skeleton (WRS) as shown in figure 3. The 


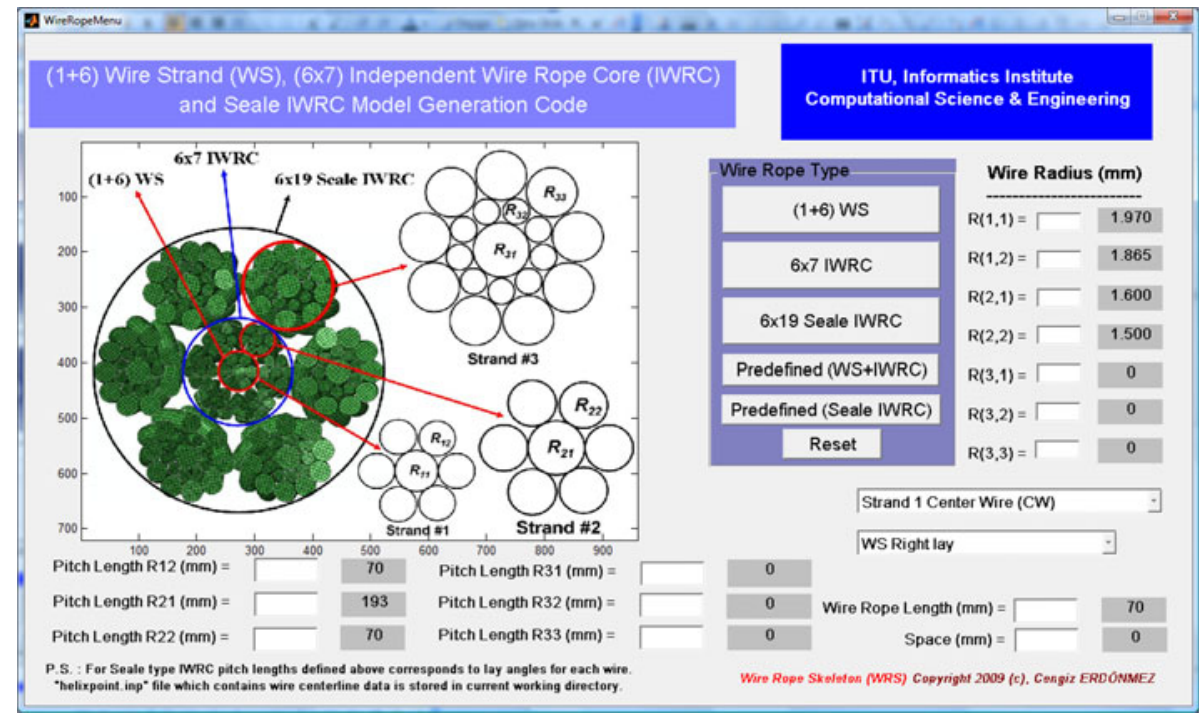

Figure 3. Wire rope skeleton (WRS), wire rope helical geometry generator code GUI.

generated helical paths are used to create the desired three-dimensional meshes of single and double helical wires of the proposed model using commercial software HyperMesh ${ }^{\mathrm{TM}}$. Helical paths used to create the outer wires of the outer strand are illustrated in figure 4.

Using the developed code and the modelling procedure both right regular lay and right lang lay IWRCs are modelled. Instead of solid geometry modelling and meshing of the wire rope, the authors offer to create meshed complete wire rope model because of the overlapping and discontinuity problems encountered during meshing and finite element analysis stages of the analysis codes. This procedure enables to construct models ready and error free for analysis purposes without length limitations. A typical meshed right lang lay IWRC model is depicted in

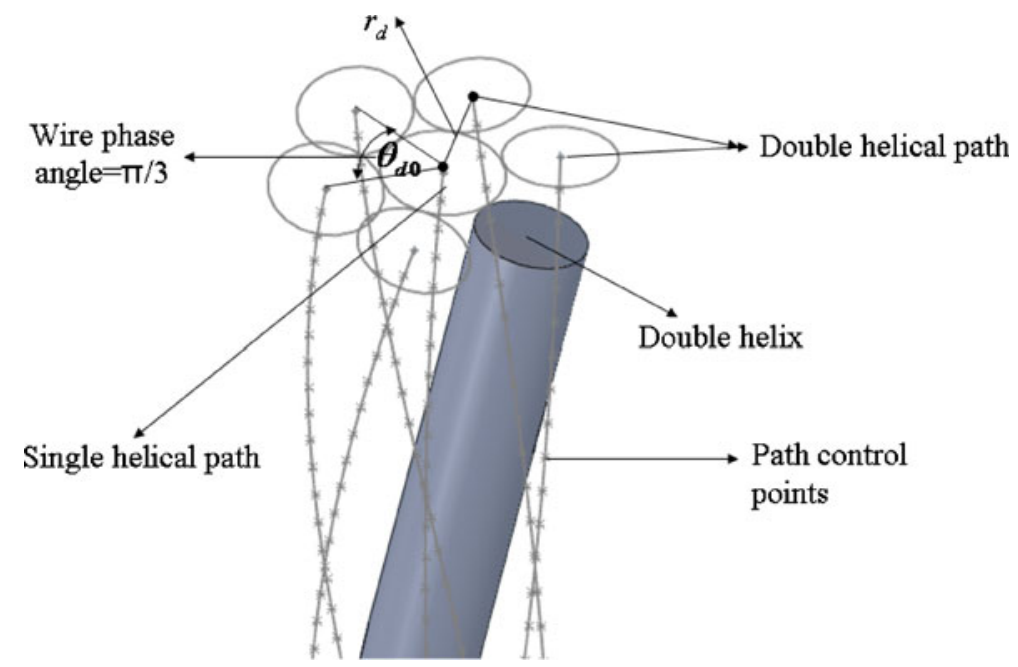

Figure 4. Double helical path illustrations. 
figure 1 which shows the accuracy of the proposed meshing using the double helical trajectory of wires within an IWRC. A cross sectional view depicted in figure 1(b) is the undeformed cross sectional view cut of the IWRC. From the figure, it can be seen easily that the cross section of a circular wire is in elliptical form. Costello (1990) considers this phenomenon in the analytical solution of the wire rope theory by using the geometrical considerations and contraction ratio of wires, which is defined as Poisson's ratio. After the wire creation procedures, the main body of the wire rope model has been assembled in finite element solver Abaqus ${ }^{\mathrm{TM}}$. Both frictionless and frictional behaviour of the simple straight strand and IWRC analysis under certain loading conditions are conducted using wire-by-wire bases with success, and the results are compared with theory and test results reported earlier.

\section{Results and discussions}

A quarter pitch length of a $28.75 \mathrm{~mm}$ length $(1+6)$ wires simple straight strand is modelled and obtained results are compared with both Costello's (1990) model and the test results reported by Utting \& Jones (1987). During the proposed numerical model, contacts between adjacent wires are defined. Elastic frictionless and elastic-plastic frictional numerical models are developed. Axial loading behaviour of a simple straight strand is investigated. An axial strain $\varepsilon$ of 0.015 , was applied in increments of 0.001 in the analysis using the displacement equivalent to the axial strain computed by $\varepsilon=(\bar{h}-h) / h$, where $h$ is the original length of the strand and $\bar{h}$ is the final length of the strand. Rotation is not allowed only in $z$ direction defined as $\Theta_{z}=0$, while the other end is constrained to be fixed boundary condition. Axial force variation with strain for the simple straight strand by pairs of comparisons is presented in figure 5. It can be seen from figure 5 that linear analytical results from Costello's solution and linear FEA results are in good agreement. For the nonlinear analysis test results of Utting \& Jones is used for comparison both with and without contact definitions. It can be seen from the results that without contact definitions resulting curve is found below the test results while the results obtained by using

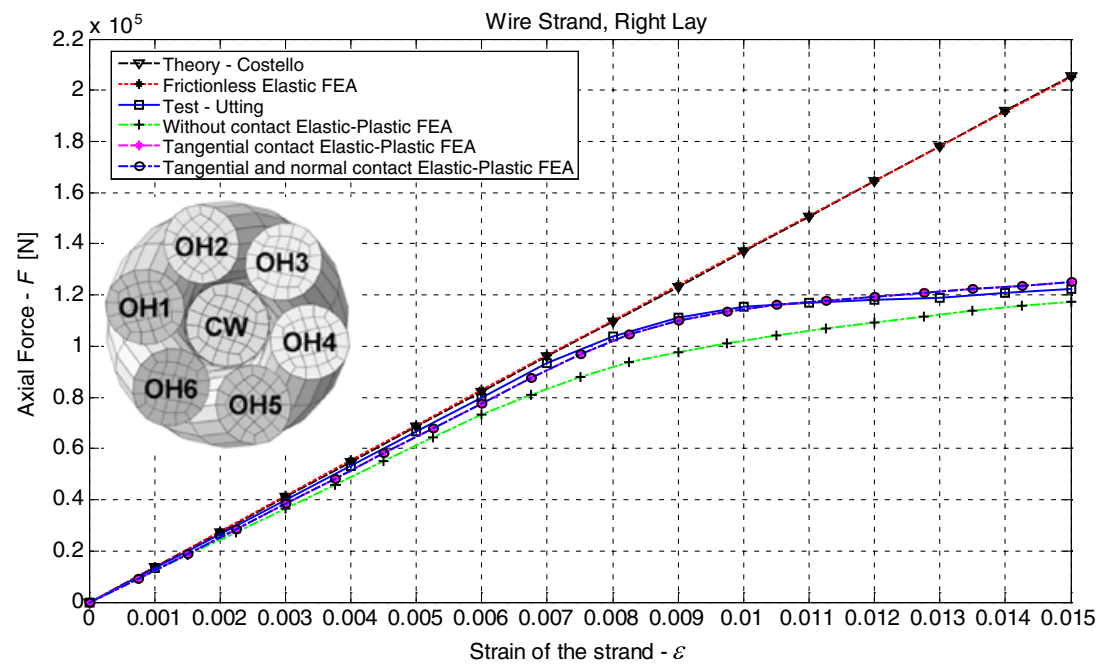

Figure 5. Force-strain results comparison; theory of Costello, test of Utting \& Jones (1987), frictionless elastic and frictional elastic-plastic FEA analysis with contact. 


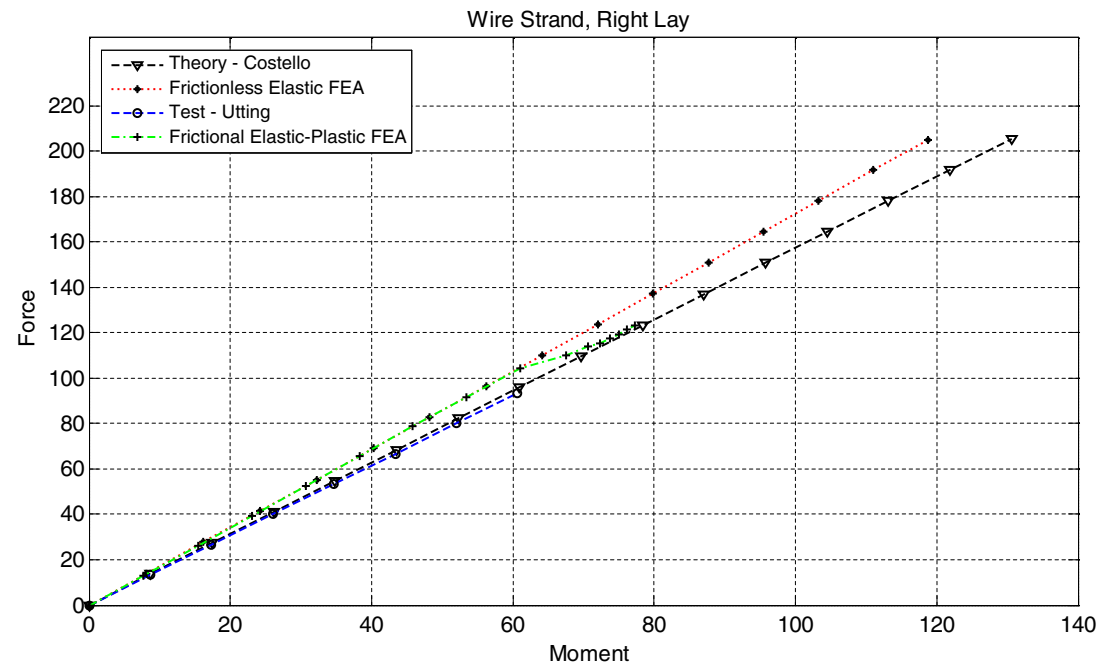

Figure 6. Force-moment results comparison; theory of Costello, test of Utting \& Jones (1987), frictionless elastic and frictional elastic-plastic FEA.

contact interactions are in good agreement with the test results. This figure shows that definition of the proposed model is working and gives accurate results.

Variation of axial force with moment is given in figure 6. One of the benefits of the current analysis method is presented in figure 7. From figure 7, wire-by-wire axial force variation with strain results can be seen and in addition, comparison with the theory of Costello is included. Wires are titled as $\mathrm{CW}$ that corresponds to center wire and $\mathrm{OH} 1-\mathrm{OH} 6$ that corresponds to outer single helical wires 1 through 6 . Center wire of the strand is loaded with $17.1 \%$ of the total axial load while an outer wire is loaded with an average $13.82 \%$ of the total axial load. This result shows that center wire carries the big portion of the axial load over the modelled straight

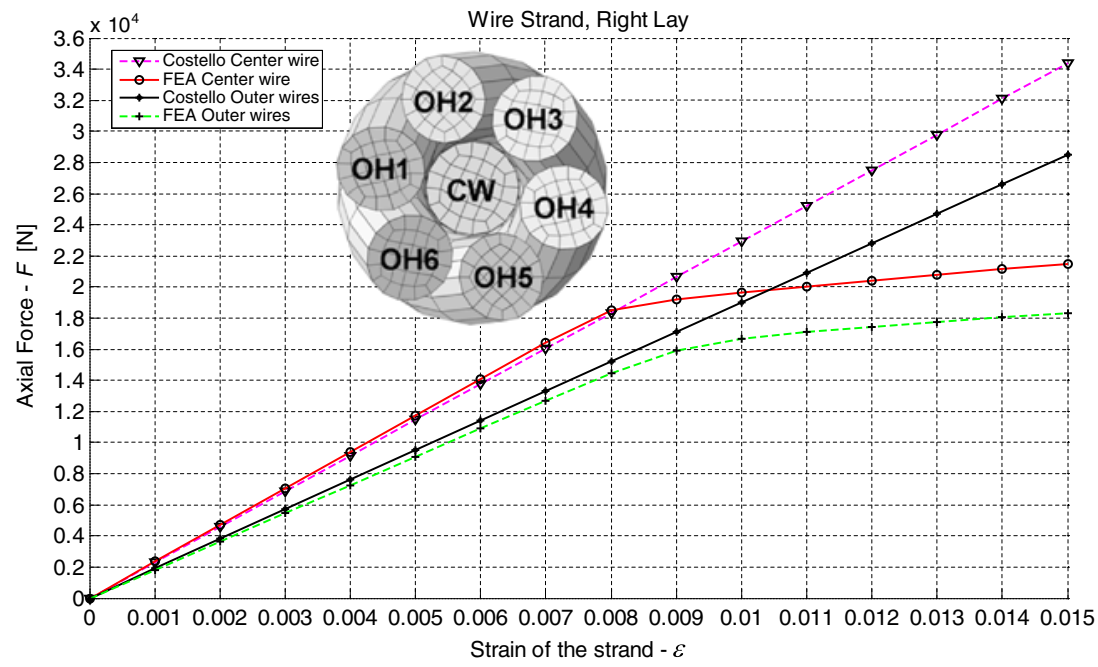

Figure 7. Wire-by-wire analysis, theory, and FEA comparison of a simple straight wire strand. 
strand. The proposed model fully accounts for frictional and plastic behaviour of the wire strand. Rotationally restrained numerical model for frictionless analysis shows reasonable agreement with the Costello's (1990) model and the frictional elastic-plastic model has in good agreement with the test results of Utting \& Jones (1987) for the application of tensile force.

The benefit of the proposed modelling and solution scheme is demonstrated in the previous application over the simple straight strand. A three-dimensional numerical model of an IWRC with $18 \mathrm{~mm}$ length is considered and FEA results are presented. IWRC's complex geometry is depicted in cross section of a meshed form in figure 1(b). Double helical geometry is taken place in the outer wires of the outer strands. Axial loading problem is solved over IWRC by applying an axial strain $\varepsilon$ of 0.015 with the increments of 0.001 . Axial force variation with strain results for each wire of the IWRC is presented using wire-by-wire based form in figure 8 . This analysis procedure shows that one can obtain detailed information from the various points using 3-D models. Core strand and outer strand wire titles are given in figure 8. A wire-by-wire loading is investigated by the analysis of a right lang lay IWRC and it has been obtained that the center wire of the core strand carries the maximum axial load. If the axial loads are compared between the core strand and the outer strand of the IWRC, core strand has loaded with an average $20.18 \%$ of the total axial load while the outer strand has loaded with an average $13.30 \%$ of the total axial load within and IWRC.

Frictionless contact controls are developed and tensile loading and twisting moments of IWRC is proposed and results are shown in figure 9. A mean axial strain, $\varepsilon$ of 0.006 , was applied in increments of 0.001 in the analysis using the displacement equivalent to the axial strain $\varepsilon$ while rotation is restrained by $\Theta_{z}=0$. Theoretical results are obtained by solving the well-known analytical model of Velinsky et al (1984) and Costello (1990) for a regular and lang lay IWRCs. The results are obtained both numerically by FEA and using theory of Costello (1990) for Poisson's ratio of $v=0$ and $v=0.3$, where wire radial contraction has been neglected and considered respectively. From the obtained results, it can be easily concluded that the wire contraction plays a very little role over the whole model analysis as discussed by Usabiaga \& Pagalday (2008). Tensile forces obtained from the proposed model have a good agreement with Costello's (1990) results for both regular lay and lang lay rope constructions. However, twisting moments give

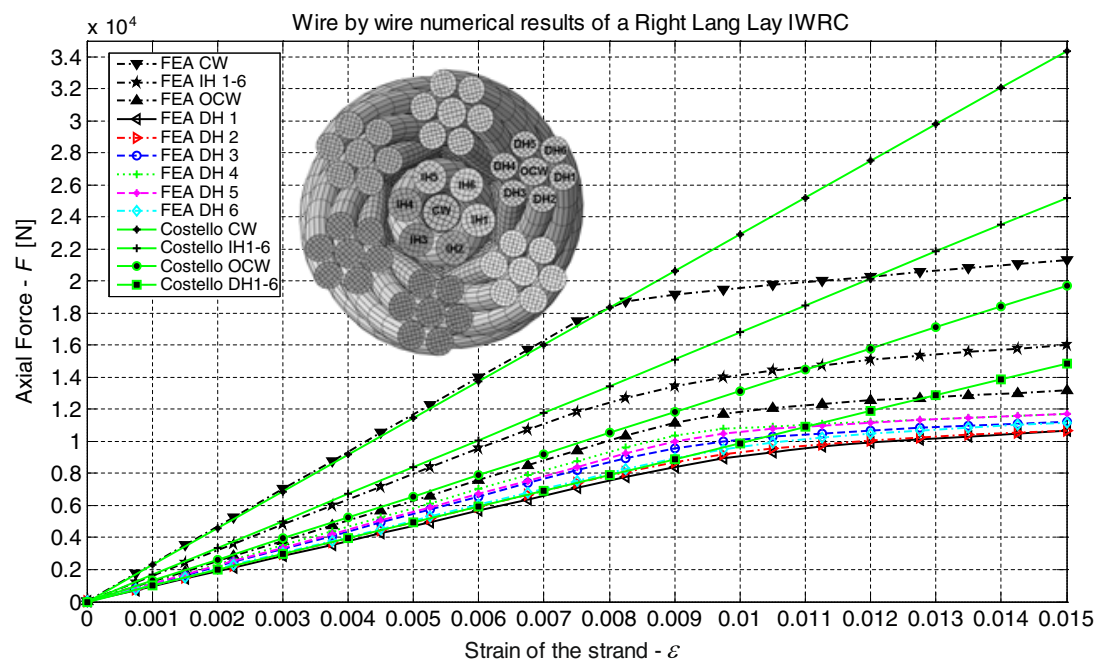

Figure 8. Wire-by-wire analysis, theory and FEA comparison of an IWRC (İmrak \& Erdönmez 2010). 

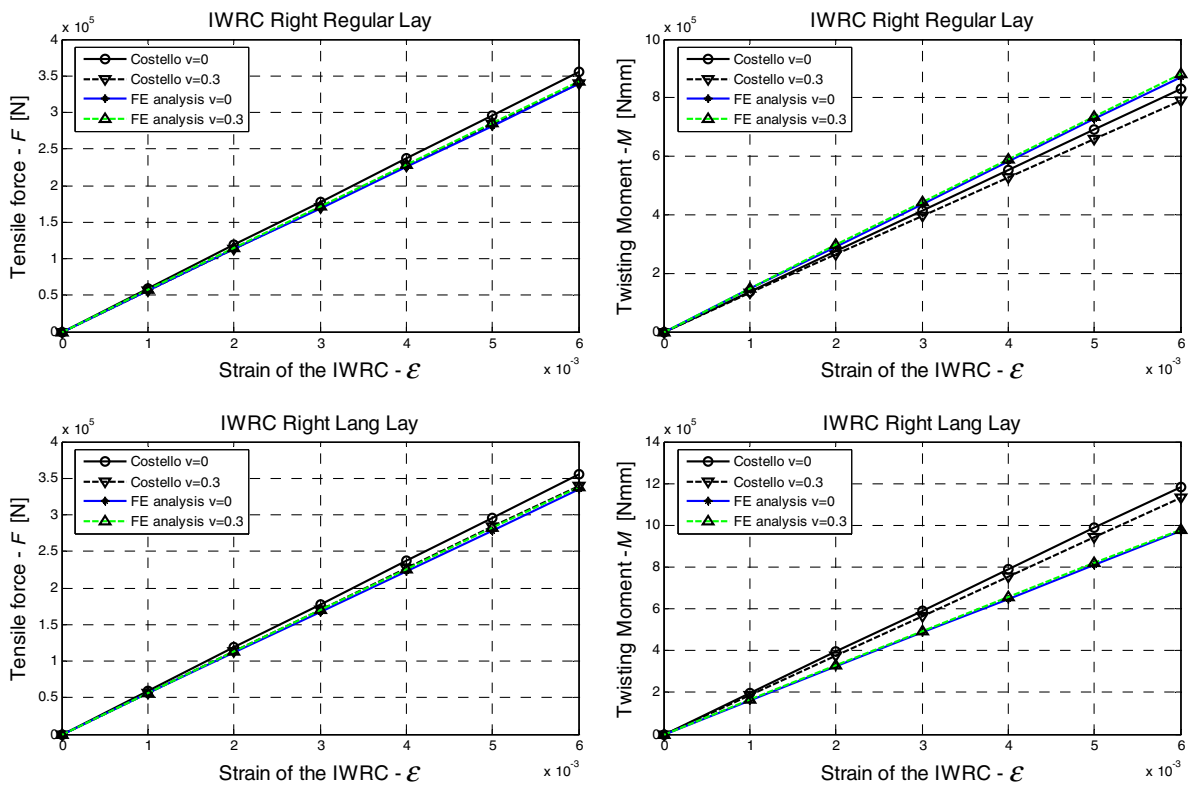

Figure 9. IWRC, Frictionless elastic, rotation restrained tensile test for $\varepsilon=0, \ldots, 0.006, \Theta_{z}=0$.

better agreement for regular lay construction than for the lang lay one when compared with Costello's (1990) results.

A frictional elastic-plastic finite element analysis is done over the same geometrical model prescribed as an $18 \mathrm{~mm}$ length $(6 \times 7)$ wire for both regular lay and lang lay IWRCs and results
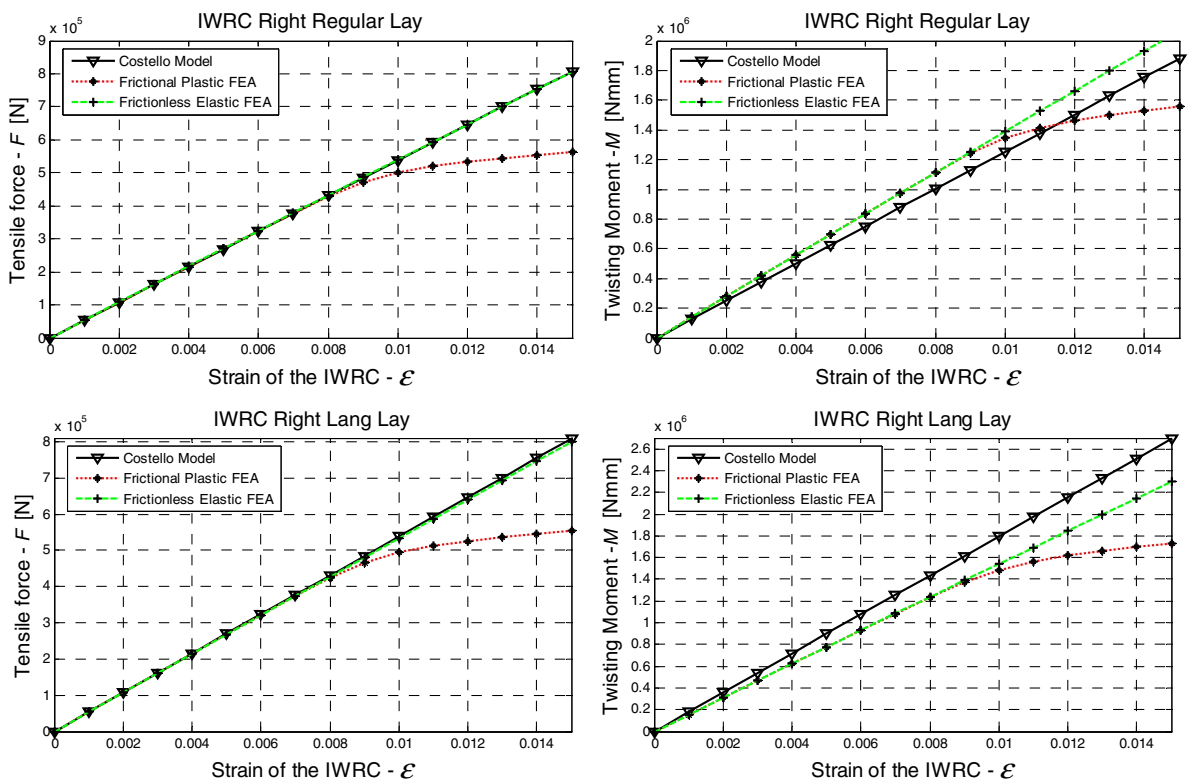

Figure 10. IWRC, Frictional elastic-plastic, rotation restrained tensile test for $\varepsilon=0, \ldots, 0.015, \Theta_{z}=0$. 


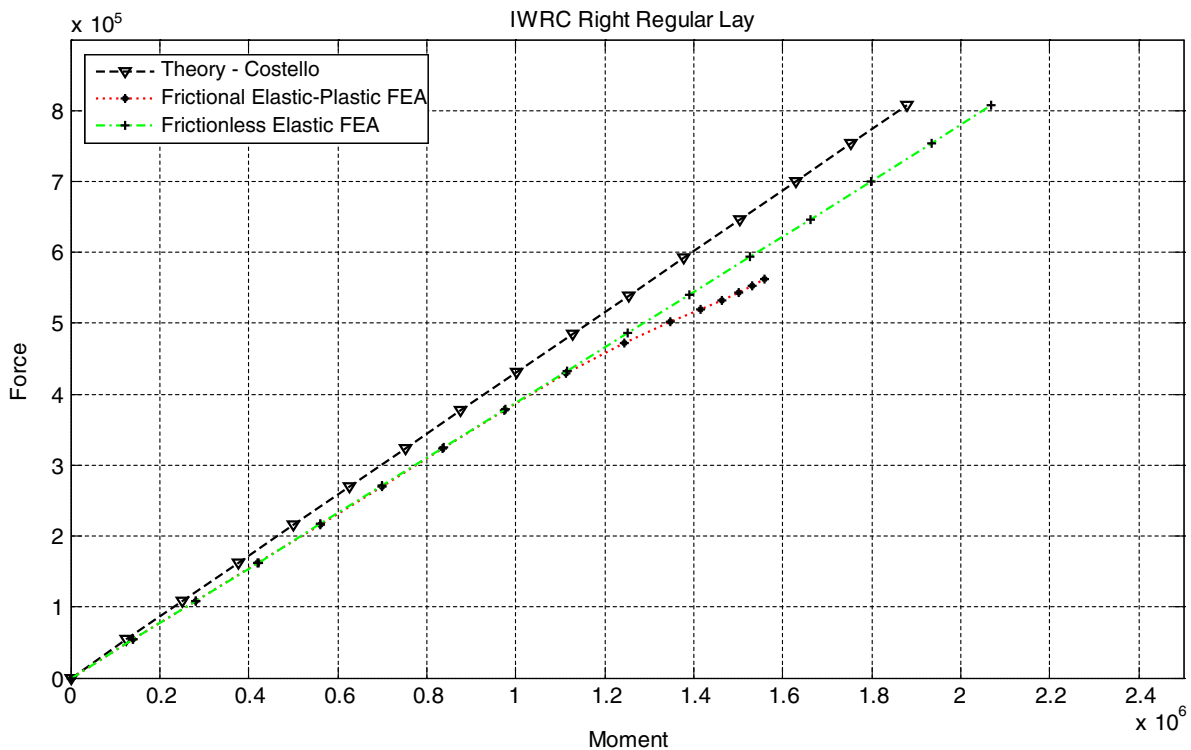

Figure 11. Force-moment results comparison of a right regular lay IWRC.

are given in figure 10. Surface to surface contact controls are developed and tensile loading and twisting moments of IWRC is proposed. Axial strain $\varepsilon$ of 0.015 , was applied in increments of 0.001 in the analysis using the displacement equivalent to the axial strain $\varepsilon$ while rotation is restrained by defining the boundary condition $\Theta_{z}=0$. Theoretical results of Velinsky et al (1984) and Costello (1990) models for a regular and lang lay IWRCs are compared with the finite

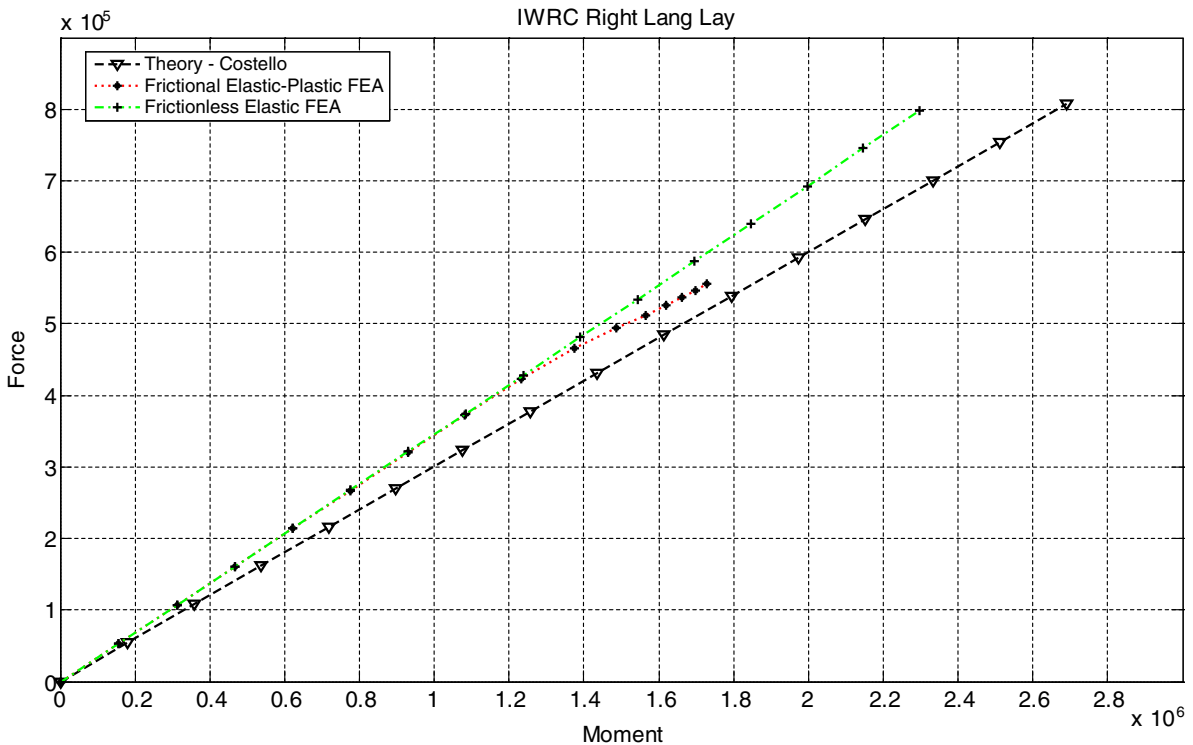

Figure 12. Force-moment results comparison of a right lang lay IWRC. 
element analysis results for the Poisson's ratio of $v=0.3$ where wire radial contraction has been considered as a realistic situation. It can be easily concluded from the figure 10 that theoretical and frictionless behaviours are in good agreement both for regular and lang lay IWRCs.

The simple straight strand analysed previously in this paper is used as the core strand of the IWRC, and the validity of the plastic behaviour of the simple straight strand has been shown in figure 5. For this reason, when the plastic behaviour of both simple straight strand given in figure 5 and IWRCs given in figure 10 are considered together, the plasticity results for both analyses show the similar behaviour. Figures 11 and 12 show the variation of axial force with moment of both right regular lay and right lang lay IWRC.

\section{Conclusion}

In this paper, a more realistic three-dimensional solid wire model of both single and double helical geometry are used while modelling and analysing IWRC. A code is developed and used while modelling wires considering the helical geometry. This modelling scheme allows one to obtain a wire rope structure without any length limitation, which can be used for more real world applications like bending and inverse bending over sheave problems. Numerical results for both frictionless elastic and frictional elastic-plastic analyses of a simple straight strand show good agreement when compared with the analytical and available test results presented in the literature. This validates the accuracy of the proposed procedure. From the presented frictionless analysis of IWRC, it can be concluded that the wire contraction plays a little role. In addition, with the 3-D numerical model wire-by-wire based analysis results can be obtained which gives more insight of the wire rope with accurate results. This enables one to analyse even each particle of a wire rope under different loading conditions by taking into account of the wires real helical geometry. The robustness of the present FE method is to give wire-bywire analysis results and enables one to obtain insights of loading through a wire rope. Proposed modelling scheme provides a chance of detailed analysis for wire ropes under various loading conditions and from this point of view, it can be used as a fundamental concept for further researches and optimization processes. Also, the proposed modelling and analysis procedure is easy to implement and use.

\section{References}

Cartraud P, Messager T 2006 Computational homogenization of periodic beam-like structures. Int. J. Solids and Struct. 43(4): 686-696

Costello G A, Sinha S K 1977 Static behaviour of wire rope. Proceedings ASCE, J. Eng. Mech. Div. 103(No.EM6): 1011-1022

Costello G A 1990 Theory of wire rope. Berlin: Springer, pp. 44-57

Elata D, Eshkenazy R, Weiss M P 2004 The mechanical behavior of a wire rope with an independent wire rope core. Int. J. Solids and Struct. 41: 1157-1172

Fekr M R, McClure G, Farzaneh M 1999 Application of ADINA to stress analysis of an optical ground wire. Computers and Struct. 72: 301-316

Ghoreishi S R, Messager T, Cartraud P, Davies P 2007 Validity and limitations of linear analytical models for steel wire strands under axial loading, using a 3D FE model. Int. J. Mech. Sci. 49: 1251-1261

İmrak C E, Erdönmez C 2010 On the problem of wire rope model generation with axial loading. Mathemat. Computational Applications 15(2): 259-268

Jiang W G, Henshall J L 1999 The analysis of termination effects in wire strand using finite element method. J. Strain Anal. 34: 1, 31-38 
Jiang W G, Henshall J L, Walton J M 2000 A concise finite element model for three-layered straight wire rope strand. Int. J. Mech. Sci. 42: 63-86

Jiang W G, Yao M S, Walton J M 1999 A concise finite element model for simple straight wire rope strand. Int. J. Mech. Sci. 41: 143-61

Jiang W-G, Warby M K, Henshall J L 2008 Statically indeterminate contacts in axially loaded wire strand. Eur. J. Mech. A/Solids 27: 69-78

Jolicoeur C, Cardou A 1991 A numerical Comparison of current mathematical models of Twisted wire cables under axisymmetric loads. J. Energy Resourc. Technol. 113: 241-249

Love A E H 1944 A treatise on the mathematical theory of elasticity. 4th ed., New York: Dover Publications, Chapter XVIII-XIX, pp. 381-426

Messager T, Cartraud P 2008 Homogenization of helical beam-like structures: application to single-walled carbon nanotubes. Computational Mech. 41(2): 335-346

Nawrocki A, Labrosse M 2000 A finite element model for simple straight wire rope strands. Computers and Struct. 77: 345-359

Phillips J W, Costello G A 1985 Analysis of wire ropes with internal-wire-rope cores. Trans. ASME 52: $510-516$

Usabiaga H, Pagalday J M 2008 Analytical procedure for modelling recursively and wire by wire stranded ropes subjected to traction and torsion loads. Int. J. Solids and Struct. 45(21): 5503-5520

Utting W S, Jones N 1987 The response of wire rope strands to axial tensile loads: Part I. and Part II. Experimental results and theoretical predictions. Int. J. Mech. Sci. 29(9): 605-619, 29(9): 621-636

Velinsky S A, Anderson G L, Costello G A 1984 Wire rope with complex cross sections. J. Eng. Mech. 110(3): 380-391

Velinsky S A 1985 General nonlinear theory for complex wire ropes. Int. J. Mech. Sci. 27: 497-507

Velinsky S A 1989 On the design of wire rope. Trans. ASME, J. Mech., Transmissions and Automation in Design 111: 382-388 